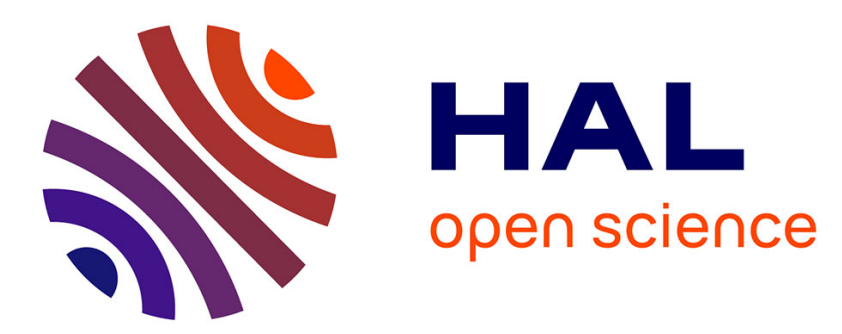

\title{
A distributed cooperative control scheme with optimal priority assignment and stability assessment
}

Haiyang Ding, Mazen Alamir, Ahmad Hably

\section{To cite this version:}

Haiyang Ding, Mazen Alamir, Ahmad Hably. A distributed cooperative control scheme with optimal priority assignment and stability assessment. IFAC WC 2014 - 19th IFAC World Congress, Aug 2014, Le Cap, South Africa. pp.11703-11708, 10.3182/20140824-6-ZA-1003.00093 . hal-00949668

\section{HAL Id: hal-00949668 \\ https://hal.science/hal-00949668}

Submitted on 24 Feb 2014

HAL is a multi-disciplinary open access archive for the deposit and dissemination of scientific research documents, whether they are published or not. The documents may come from teaching and research institutions in France or abroad, or from public or private research centers.
L'archive ouverte pluridisciplinaire HAL, est destinée au dépôt et à la diffusion de documents scientifiques de niveau recherche, publiés ou non, émanant des établissements d'enseignement et de recherche français ou étrangers, des laboratoires publics ou privés. 


\title{
A distributed cooperative control scheme with optimal priority assignment and stability assessment
}

\author{
Haiyang DING, Mazen ALAMIR, ${ }^{*}$ Ahmad HABLY* \\ * University of Grenoble, Control Systems Departement Rue de la \\ Houille Blanche, Domaine Universitaire, Saint Martin dHeres, France, \\ email: \{haiyang.ding, mazen.alamir, ahmad.hably\}@gipsa-lab.fr
}

\begin{abstract}
In this paper, a distributed partially cooperative control framework is proposed for a network of linear interconnected subsystems. It is assumed that each subsystem in the network possesses its own objective and a corresponding nominal interaction-free state feedback law. The proposed framework enables each subsystem to compute an additional control term in order to help maintaining the integrity of the overall network. As this cooperation-like behavior involves relative priority assignment, a communication aware heuristic is proposed with an associated stability assessment that is based on the closed-loop network matrix's spectrum monitoring. Illustrative examples are used to assess the effectiveness of the proposed scheme including a distributed load frequency problem.
\end{abstract}

\section{INTRODUCTION}

The design of distributed control framework for interconnected systems has received a great attention in the last recent years due to the fact that centralized frameworks are often very expensive in terms of communication if not impossible to be settled. On the other hand, when the interactions between subsystems are strong, the fully decentralized control schemes show bad performance Ho [2005], Cui and Jacobsen [2002]. To overcome these limits, distributed control schemes have been proposed. The main idea is that each subsystem of the network has to cooperate with its neighbors by sharing partial information in order to contribute to the global objective of the network (or to manage its own objective) while avoiding destabilizing the whole network.

The literature related to the distributed/cooperative control of interconnected networks has witnessed an impressive growth these last years. This makes quite difficult an exhaustive relative positioning of the existing propositions. An efficient way towards a comprehensive positioning is to consider the control paradigm being addressed, the information shared between network's subsystems and the coupling between them, and at the end the stability enhancement and assessment.

- In Dunbar and Murray [2006], Richards and How [2004a], Franco et al. [2008], the paradigm lies in performing a distributed solution of a unified optimal control problem that involves all the subsystems.

- The amount of information items that is communicated or shared among the subsystems is another important issue in distributed control design. In T. Keviczky and Hrovat [2006], Brett T. Stewart [2010], the dynamic models of all neighbors are completely shared while in Dunbar [2007], A.N. Venkat and Wright [2008], Richards and How [2004a], the whole states and control vector and/or trajectories are transmitted.

- The coupling between subsystems in the networks can be limited to terms involving only control vectors (Camponogara and de Oliveira [2009]), state vectors (Jia and Krogh [2001], E. Camponogara and Talukdar [2002], Dunbar [2007]) or general coupling terms that do not induce instability (Dunbar and Caveney [2012], Richards and How [2004b], T. Keviczky and Hrovat [2006]).

- The stability assessment results can be obtained by monitoring the spectrum of the closed-loop network's matrix either through LMI design or by checking the eigenvalues (Michel and Miller [1985], Jia and Krogh [2001], E. Camponogara and Talukdar [2002], T. Li and Fei [2010]).

In the present work, we consider linear networks involving general potentially destabilizing interconnection terms that involve both states and control. It is assumed that each subsystem has its own objective, its own nominal feedback law and its own Lyapunov function. This paradigm is industrially relevant as practitioners often prefer rather modular design framework in which networkrelated issues and solutions are added as an additional layer that does not question the old existing widely assessed local controllers. The information exchanged between subsystems preserves the details of the local decisions and set-ups from being totally exchanged and only aggregated quantities are blindly transmitted as far as this transmission helps preserving the stability of the overall network. Such kind of parsimonious information exchange has been already adopted in (Farina and Scattolini [2012], Namerikawa and Kato [2011], Richards and J.How [2007]) where only the interaction-related terms are exchanged between neighbors. For the stability assessment, some priority coefficients optimized off-line have been used. More precisely, for a given set of these priority coefficients, the 
controls at the subsystems' level are completely defined. Only once these coefficients have been successively optimized by the network's control designer who has the complete knowledge of the overall network then the distributed cooperative control scheme stability can be guaranteed.

The paper is organized as follows. In section 2, the control problem is stated and the network equations and related assumptions are given. In section 3, the distributed cooperative control scheme is progressively introduced highlighting the role of the priority matrix. More precisely, it is shown how each subsystem in the network constructs an optimal control problem involving both its own objective and the stability-related concerns of its neighbors. The latter are transmitted through dedicated exchanged items. The way terms coming from neighbors impacts the local optimal control problem heavily depends on the priority of the neighbors as viewed by the local subsystems. The way the priority matrix is optimized using off-line dedicated nonlinear programming is detailed in section 4 . Section 5 shows the effectiveness of the proposed scheme with stability assessment using two examples including a well known frequency distributed control problem in order to illustrate the different features of the proposed framework and to assess its efficiency in yielding communication-aware stabilizing cooperative control scheme. Finally section 6 concludes the paper and gives hints for further investigation. The following notations are used throughout the paper:

- $N$ : total number of subsystems in the network

- $N_{j}$ : number of systems that are affected by system $j$

- $n_{i}^{u}$ and $n_{i}$ : dimension of input and state vector of subsystem $i$

- $\mathbb{O}$ : array with only zero elements of appropriate dimensions

- $\rightarrow$ and $\leftarrow$ indicate which system is affecting the other. For example, a variable with $(j \rightarrow i)$ indicates that this variables is involved in the way subsystem $j$ influences subsystem $i$

- $\longleftrightarrow$ and $\longleftarrow$ indicate the direction of the information circulation. For example, a variable with $(j \longmapsto i)$ indicates that it is sent from subsystem $j$ to subsystem $i$

- $\mathcal{I}_{j}$ : A set containing indices of subsystems that are affected by subsystem $j$

- $\mathcal{I}_{j}^{\leftarrow}$ : A set containing indices of subsystems that affect system $j$

\section{PROBLEM STATEMENT AT A SUBSYSTEM LEVEL}

Let us consider an interconnected network in which a subsystem $i$ is governed by the following Ordinary Differential Equation (ODE):

$$
\dot{x_{i}}=A_{i} x_{i}+B_{i} u_{i}+\sum_{j \in \mathcal{I}_{i}^{\leftarrow}}\left(A_{j \rightarrow i} x_{j}+B_{j \rightarrow i} u_{j}\right)
$$

where $x_{i}, u_{i}$ are respectively the state and the control vector of subsystem $i$ while the summation term describes the effects of interaction with neighbors on subsystem $i$. Based on the above argument, it is assumed that subsystem $i$ can be stabilized by a local controller: $u_{i}=$ $-K_{i} x_{i}$ such that there are a Lyapunov function $V_{i}\left(x_{i}\right)=$
$x_{i}^{T} P_{i} x_{i}$ and some positive definite function $S\left(x_{i}\right)$ such that the following inequality holds in the nominal case:

$$
\left.\dot{V}_{i}\left(x_{i}\right)\right|_{\dot{x}_{i}=A_{i}^{c l} x_{i}} \leq-S_{i}\left(x_{i}\right)
$$

where $P_{i}$ is a symmetric positive definite matrix and $A_{i}^{c l}=A_{i}-B_{i} K_{i}$ is used to denote the nominal closedloop matrix of subsystem $i$.

When interaction with other subsystems is present, a cooperative control term $v_{i}$ should be designed and added to the nominal term by which subsystem $i$ can keep its own objective while helping its neighbors to maintain the global stability. Thus,

$$
u_{i}=-K_{i} x_{i}+v_{i}
$$

Consequently, equation (1) can be rewritten in the following more compact form:

$$
\dot{x}_{i}=A_{i}^{c l} x_{i}+\sum_{j \in \mathcal{I}_{i}^{\leftarrow}}\left(A_{j \rightarrow i} x_{j}+B_{j \rightarrow i} u_{j}\right)
$$

in which $i \in \mathcal{I}_{i}^{\leftarrow}$ meaning simply that subsystem $i$ is impacting itself. This convention is used in the reminder of the paper. Once the interaction is taken into account, the inequality (2) becomes:

$$
\dot{V}_{i}\left(x_{i}\right) \leq-S_{i}\left(x_{i}\right)+\sum_{j \in \mathcal{I}_{i}^{\leftarrow}} L^{j \rightarrow i}\left(x_{i}\right) \cdot\left(A_{j \rightarrow i} x_{j}+B_{j \rightarrow i} u_{j}\right)
$$

where $L^{j \rightarrow i}\left(x_{i}\right)=2 x_{i}^{T} P_{i}$. Based on the above expressions, one has the following straightforward lemma:

\section{Lemma 1. [Stability constraint for subsystem $i$ ]}

The subsystem $i$ is asymptotically stable provided that the following inequality holds:

$$
\sum_{j \in \mathcal{I}_{i}^{\leftarrow}} L^{j \rightarrow i}\left(x_{i}\right) \cdot\left(A_{j \rightarrow i} x_{j}+B_{j \rightarrow i} u_{j}\right)<\mu S_{i}\left(x_{i}\right)
$$

for some $\mu \in[0,1[$.

Now this requirement has to be sent, in a rather condensed form to all the neighbors affecting $i$ (namely, having indices $j \in \mathcal{I}_{i}^{\leftarrow}$ ). For this reason, the following assumption is needed regarding communication rules as well as mutual a priori knowledge available at each subsystem regarding the neighbors characteristics:

\section{Assumption 1. [Knowledge/Communication rule \& Network topology]}

(1) The structure of interaction in the network is bidirectional, namely:

$$
\left\{i \in \mathcal{I}_{j}^{\leftarrow}\right\} \Longleftrightarrow\left\{j \in \mathcal{I}_{i}^{\leftarrow}\right\}
$$

(2) Each subsystem $j$ that affects $i\left(j \in \mathcal{I}_{i}^{\leftarrow}\right)$ is aware of the interaction matrices $A_{j \rightarrow i}$ and $B_{j \rightarrow i}$ involved in (4).

(3) At each time step, subsystem $i$ sends to its neighbors $j \in \mathcal{I}_{i}^{\leftarrow}$ (affecting it) the following quantity:

$$
W_{i \longmapsto j}:=L^{j \rightarrow i}\left(x_{i}\right)
$$

It should be pointed out that each subsystem $j(j \neq i)$ neither knows the dynamics of $W_{i \hookrightarrow j}$ nor the explicit value of the state $x_{i}$ for all $i$ that is affected by it $\left(i \in \mathcal{I}_{j}\right)$. The information being sent concerns the instantaneous value of $L^{j \rightarrow i}\left(x_{i}\right)$. Note however that as soon as the subsystem $j$ 
receives the value of $W_{i \longmapsto j}$, it can consider the left hand side of (6) as a linear combination of $x_{j}$ and $u_{j}$ that has to be reduced if subsystem $i$ needs to be stabilized. But since there are as many subsystems $i$ as there are elements in the set of indices $\mathcal{I}_{j}$, there is clearly need to define a relative priority weighting viewed by subsystem $j$. This is the aim of the following section.

\subsection{The priority vector}

In many networks, some subsystems are more critical than others. In such situations, it seems reasonable to consider the stability constraints sent by such critical subsystems with higher weight (or priority) that the stability constraints sent by other less critical subsystems. Since only neighbors belonging to $\mathcal{I}_{j} \rightarrow$ are considered by $j$, the relative priority weights are intimately linked to the connection topology. To better explain this feature, let us consider the following example where a network with 4 subsystems coupled in a chain graph as shown in the right drawing of Fig. 1. An associated priority matrix can be given in the
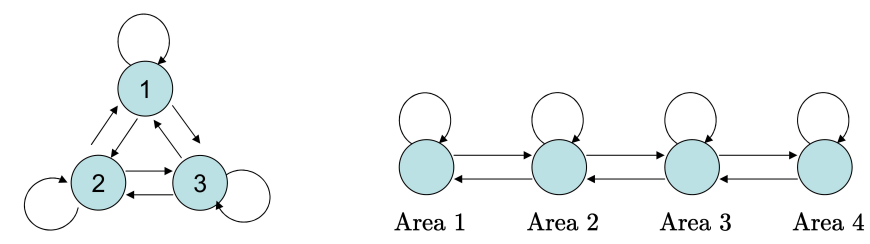

Fig. 1. Example of a coupled networks

following form:

$$
\Pi=\left[\begin{array}{llll}
\pi_{1} & \pi_{2} & \pi_{3} & \pi_{4}
\end{array}\right]=\left[\begin{array}{cccc}
\pi_{1}^{1} & \pi_{2}^{1} & 0 & 0 \\
\pi_{1}^{2} & \pi_{2}^{2} & \pi_{3}^{2} & 0 \\
0 & \pi_{2}^{3} & \pi_{3}^{3} & \pi_{4}^{3} \\
0 & 0 & \pi_{3}^{4} & \pi_{4}^{4}
\end{array}\right] \quad ; \quad \pi_{i}^{j} \geq 0
$$

In (8), $\Pi$ and $\pi_{j}$ denote respectively the priority matrix of the network and the priority vector of subsystem $j$. $\pi_{i}^{j}$ determines how important subsystem $i$ is viewed by subsystem $j$. Roughly speaking, the higher $\pi_{i}^{j}$ is, the more important subsystem $i$ is considered by subsystem $j$.

On receiving the information $W_{i \longmapsto j}$ subsystem $j$ defines a cost function to be minimized based on a given priority vector in order to cooperate with all subsystems having index $i \in \mathcal{I}_{j}$. Namely

$$
\begin{array}{r}
\min _{v_{j}}\left\{\left\|v_{j}\right\|_{R_{j}}^{2}+\sum_{i \in \mathcal{I}_{j}} \pi_{j}^{i}\left[W _ { i \longmapsto j } \left(A_{j \rightarrow i} x_{j}+\right.\right.\right. \\
\left.\left.\left.B_{j \rightarrow i}\left(-K_{j} x_{j}+v_{j}\right)\right)\right]\right\}
\end{array}
$$

Note that the above optimization problem is defined given a pre-assigned value of the priority vector $\pi_{j}$.

\section{SOLVING THE OPTIMIZATION PROBLEM (9)}

In order to solve (9) and derive the additional control term $v_{j}$, an extended dynamic system is constructed:

$$
\dot{z}_{j}=\mathcal{A}_{j} z_{j}+\mathcal{B}_{j} v_{j}=\left[\begin{array}{ccc}
A_{j}^{c l} & G_{j}^{1} & \ldots \\
\mathbb{O} & G_{j}^{N_{j}}
\end{array}\right] z_{j}+\mathcal{B}_{j} v_{j}
$$

where

$$
z_{j}:=\left(\begin{array}{c}
x_{j} \\
\pi_{j}^{1} W_{1 \longmapsto j}^{T} \\
\vdots \\
\pi_{j}^{N_{j}} W_{N_{j} \rightarrow j}^{T}
\end{array}\right), \mathcal{B}_{j}=\left(\begin{array}{c}
B_{j} \\
\mathbb{O}
\end{array}\right), \Gamma=\operatorname{diag}(-\epsilon)
$$

with $\epsilon$ is a positive constant, $G_{j}^{i}=\frac{1}{2 \pi_{j}^{i}} \cdot\left(A_{i \rightarrow j}-B_{i \rightarrow j} K_{i}\right)$. $\left(P_{i}\right)^{-1}$ while assuming a hypothetical exponentially decaying dynamic of $W_{i \succ j}^{T}{ }^{1}$.

\subsection{Rewriting of the cost function}

By expressing $\pi_{j}^{i} W_{i \longmapsto j}$ and $x_{j}$ in terms of the extended state $z_{j}$, and by using the following assumption:

\section{Assumption 2. [Shared Knowledge]}

For any subsystem $i$ that are affected by $j\left(i \in \mathcal{I}_{j}\right)$, subsystem $j$ knows the matrix

$$
\left(A_{i \rightarrow j}-B_{i \rightarrow j} K_{i}\right) \cdot\left(P_{i}\right)^{-1} \in \mathbb{R}^{n_{j} \times n_{i}}
$$

The optimization problem (9) can be put in the following form:

$$
\min _{v_{j}}\left\{v_{j}^{T} R_{j} v_{j}+z_{j}^{T} Q_{j}^{c o o p} z_{j}+z_{j}^{T} N_{j}^{c o o p} v_{j}\right\}
$$

with

and

$$
Q_{j}^{\text {coop }}=\left(\begin{array}{cccc}
0 & A_{j \rightarrow 1} & \ldots & A_{j \rightarrow N_{j}} \\
-B_{j \rightarrow 1} K_{j} & 0 & \ldots & 0 \\
\vdots & 0 & \ldots & 0 \\
-B_{j \rightarrow N_{j} \rightarrow K_{j}} & 0 & \ldots & 0
\end{array}\right)
$$

$$
N_{j}^{c o o p}=\left(\begin{array}{c}
\mathbb{O}_{n * n_{u}} \\
B_{j \rightarrow 1} \\
\vdots \\
B_{j \rightarrow N_{j}}
\end{array}\right)
$$

By now, the linear dynamic model (10) and the quadratic cost function (11) completely define a quadratic programming problem that can be solved in a discrete-time setting to compute the matrix gain $K_{j}^{v}$ that is used by subsystem $j$ in order to compute the additional control $v_{j}$ according to:

$$
v_{j}=-K_{j}^{v} z_{j}
$$

The use of sampling control here enables the non necessarily negligible communication time to be taken into account.

Note that the above optimization problem is defined given a pre-assigned value of the priority vector $\pi_{j}$. In section 4 , it is shown how the priority vectors can be computed such that the resulting overall interconnected cooperative network is asymptotically stable. However, let us first show how (9) can be solved at the subsystem $j$ level.

\section{STABILITY ENHANCEMENT THROUGH THE OPTIMIZATION OF THE PRIORITY VECTORS}

The preceding sections propose a heuristic that results in the complete definition of the distributed feedback control

\footnotetext{
1 The derivation is presented in the appendix
} 
once given a priority matrix $\Pi$ [see (8)]. In other words, regardless how justified is the heuristic proposed above it leads to a rigorously defined terms $v_{j}, j \in\{1, \ldots, N\}$ leading to rigorously defined controls $u_{j}, j \in\{1, \ldots, N\}$. In this section, it is shown that based on these resulting control expressions (issued from heuristically defined procedure), one can rigorously compute the spectrum of the network's dynamic closed loop matrix as a function of the priority matrix $\Pi$. Therefore, $\Pi$ can be optimized by the network control designer (in the off-line design step) in order to guarantee the stability of the network. This is explained in the following sections.

\subsection{The complete state space model}

A complete state space model of the distributed scheme is built in which the states of all the subsystems are involved. The rationale behind this is that although $W_{i \rightarrow j}$ is known to subsystem $j$ as an information sent by subsystem $i$, it is known from a designer's view as:

$$
W_{i \longmapsto j}=L^{j \rightarrow i}\left(x_{i}\right)=2 x_{i}^{T} P_{i}
$$

Since $W_{i \longmapsto j}$ is involved in the extended state $z_{j}$ and (13) is linear in $x_{i}, z_{j}$ can be expressed as linear combination of the state vectors $\left\{x_{i} \mid i \in \mathcal{I}_{j}\right\}$. Once all the extended states for each subsystem in the network are expressed in this manner, the complete state space model that is equivalent to the distributed scheme can be established. This results in the following compact autonomous form:

$$
X^{+}=\mathbb{E}(\Pi) X
$$

with $X=\left[\begin{array}{llll}x_{1}{ }^{T} & x_{2}{ }^{T} & \cdots & x_{N}^{T}\end{array}\right]^{T}$ in which $\Pi$ is the priority matrix defined above.

\subsection{Stability analysis and off-line optimization of the priority matrix $\Pi$}

Let us use the following notation in order to refer to the spectrum of the network matrix for a given priority vector $\Pi$ :

$$
\alpha(\Pi)=\max _{i}\left|\lambda_{i}(\mathbb{E}(\Pi))\right|
$$

where $\lambda_{i}$ is the $i$-th eigenvalue of $\mathbb{E}(\Pi)$. Therefore, the stability of the distributed control based on the heuristic explained in the preceding sections can be guaranteed as soon as a priority matrix $\Pi$ can be found such that $\alpha(\Pi)<1$. Note also that $\mathbb{E}(\Pi)$ can be written in the following form:

$$
\mathbb{E}(\Pi)=\bar{\pi}_{1} E_{1}+\cdots+\bar{\pi}_{N_{p}} E_{N_{p}}
$$

in which $N_{p}$ is the number of nonzero elements in the connection matrix $C$ while $\bar{\pi}_{i}$ 's are the corresponding elements of the priority matrix. The problem of minimizing the radius of the spectrum of (16) is a well known problem in the matrix-pencil literature (Burke et al. [2000]). Several methods can be used to minimize the radius $\alpha(\Pi)$ :

$$
\min _{\Pi \in \mathcal{P}}\{\alpha(\Pi)\}
$$

where $\mathcal{P} \in \mathbb{R}^{N_{p}}$ is a compact subset of admissible values to defined according to the context. In the sequel, the MATLAB subroutine FMINCON is used to solve the above optimization problem leading to the optimal priority matrix $\widehat{\Pi}$.
Remark 2. When considering a large scale network, the problem of defining the form of the priority matrix is important since the matrix pencil optimization with high dimension is hard to perform. One potential solution is to reduce the number of degrees of freedom in the definition of the priority matrix by using reduced dimensional parametrization of the form $\Pi=\Pi(\rho)$. The dimension of the optimization problem is therefore defined by the dimension of the parameter vector $\rho$.

\section{ILLUSTRATIVE EXAMPLES}

In this section, some illustrative examples are given to show the efficiency of the proposed framework. In all the examples, evolutions labeled with coop refer to the distributed cooperative method while those labeled with noncoop correspond to scenarios where the distributed cooperative control is not activated (i.e. $u_{i}=-K_{i} x_{i}$; $\left.v_{i}=0\right)$.

\subsection{Example 1: Stability enhancement of a numerical case study}

In this first example, a network is built randomly using 3 subsystems with 2 states and 1 control each. The subsystems are interconnected with each other in a cyclic graph as in Fig. 1 through states and control inputs. The continuous-time equation of the subsystems are given as follows:

$$
\dot{x}_{i}=A_{i} x_{i}+B_{i} u_{i}+\beta \sum_{j \in \mathcal{I}_{i}^{\leftarrow}}\left(A_{j \rightarrow i} x_{j}+B_{j \rightarrow i} u_{j}\right)
$$

in which $A_{i}$ is the open-loop state matrix governing subsystem $i$. The nominal local controllers are designed with LQR method: $A_{i}^{c l}=A_{i}-B_{i} K_{i}$. The cooperative control is computed as presented in the previous sections. Note that $\beta$ expresses the strength of the coupling between subsystems. The values of the matrices can be found in the appendix. At each $\beta$, three different control schemes are applied to the resulted network: (a) only nominal decentralized controllers are activated, (b) the cooperative control with equal priority and (c) the cooperative control with optimal priority.

In the cooperative control with equal priority, all the priority vectors are set to $\pi_{i}=\left[\begin{array}{lll}1 & 1 & 1\end{array}\right]^{T}$ for each subsystem. The optimal priority is obtained using the method described in section 4 . The radiuses of the spectrum $\alpha(\Pi)$ of the three complete state space models when $\beta$ grows from 0 are shown in Fig. 2.

Fig. 3 is a zoom-in of Fig. 2. The zoom-in figure shows that nominal decentralized control can stabilize the network when $\beta<1.62$. Beyond this value, subsystems with cooperation (using equal priorities) preserves the network stability as far as $\beta<1.82$. The figure on the right shows that the cooperative control with optimal priority is able to stabilize the subsystems until $\beta$ reaches 15 . The fluctuations of curve is due to the stopping criterion of the algorithm.

\subsection{Example 2: Load Frequency control problem}

In this example, the distributed control scheme is applied to the load frequency control problem described in (Namerikawa and Kato [2011]). The network consists of 


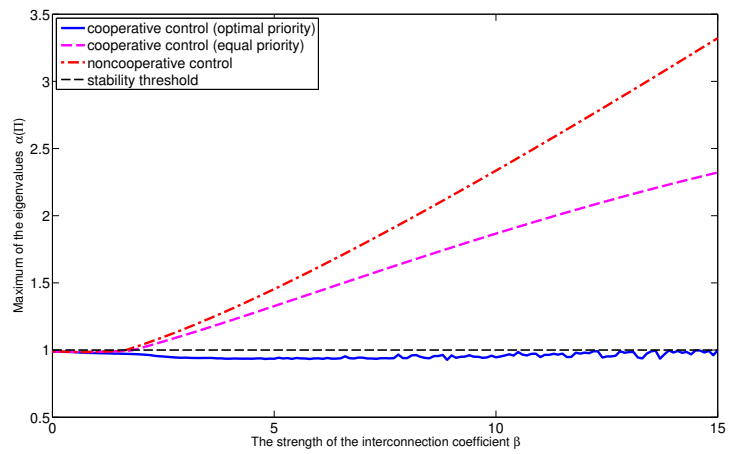

Fig. 2. Example 1: The evolution of the spectrum of the network under growing interconnection strength.

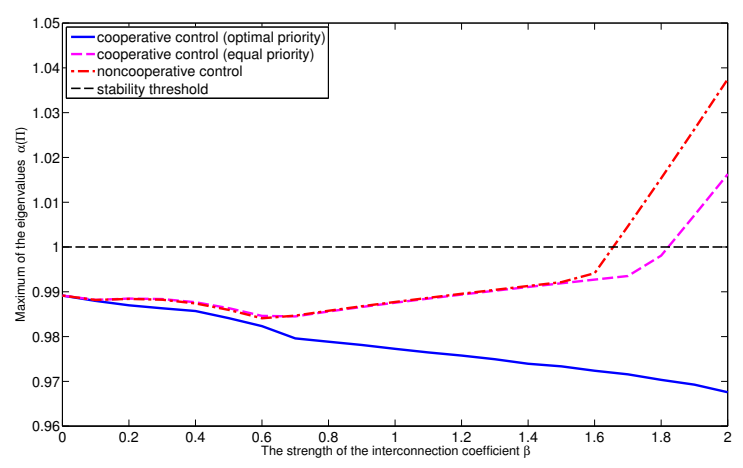

Fig. 3. Example 1: A zoom-in of the previous figure.

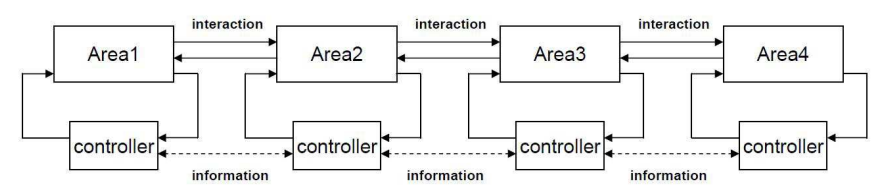

Fig. 4. The structure of the power network

$N=4$ subsystems (areas) and each of them has $n=7$ states which are coupled in a chain-like graph (see Fig. 4). The parameters of the network are taken according to (Namerikawa and Kato [2011]) and the states of each area are influenced by a white noise with covariance matrix $10^{-3} \times \mathbb{I}$. An additional coefficient $\beta$ is added to tune the strength of coupling between areas.

Four case studies are presented to illustrate the advantages of the proposed scheme. The equations of the network are given in appendix $\mathrm{C}$.

Case study 1 - Cooperative control with equal priority: In the first simulation, the coupling parameter $\beta=1$ is used (leading to the same model as (Namerikawa and Kato [2011])). When the cooperative control is not activated, the network spectrum radius is 0.99 which means that the network is stable under the decentralized nominal control. Nevertheless, when looking at the behaviors of the Lyapunov function of the cooperative control $V_{\text {coop }}$ and that of the decentralized nominal control $V_{\text {noncoop }}$, it can be seen on Fig. 5 that the firing of the cooperation algorithm leads to far better overall performance.

Note that for the purpose of better illustrating the evolution of the Lyapunov functions (which we choose to evaluate the performances of the areas), the initial conditions for all states of the ares are set to 1 (since all of them are stable).

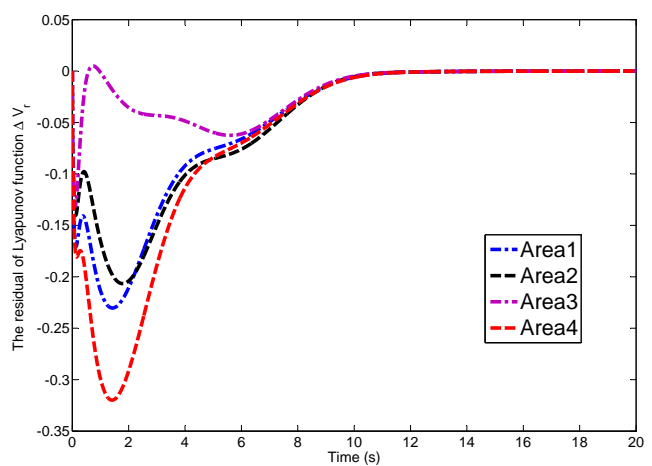

Fig. 5. Example 2 - case study 1: Evolution of $\Delta V_{r}=$ $V_{\text {coop }}-V_{\text {noncoop }}$ indicating that cooperation with equal priority enhance the overall performance of the network even when decentralized control is sufficient to achieve the stability (weak coupling).

Case study 2 - Cooperative control with higher priority on certain subsystem: In this simulation the coupling parameter is also chosen as $\beta=1$, and we apply the priority matrix defined by:

$$
\Pi=\left(\begin{array}{cccc}
10 & 10 & 0 & 0 \\
1 & 1 & 1 & 0 \\
0 & 1 & 1 & 1 \\
0 & 0 & 1 & 1
\end{array}\right)
$$

which means that Area 1 is given a higher priority viewed by both itself and its neighbor Area 2 . The behavior of the Lyapunov function $V_{\text {coopAreal }}$ is compared to that of the cooperative control with equal priority $V_{\text {coop }}$. One can see form Fig. 6 that the behavior of Area 1 is improved while for other areas, since they are defined to be less important, their performances are a little sacrificed.

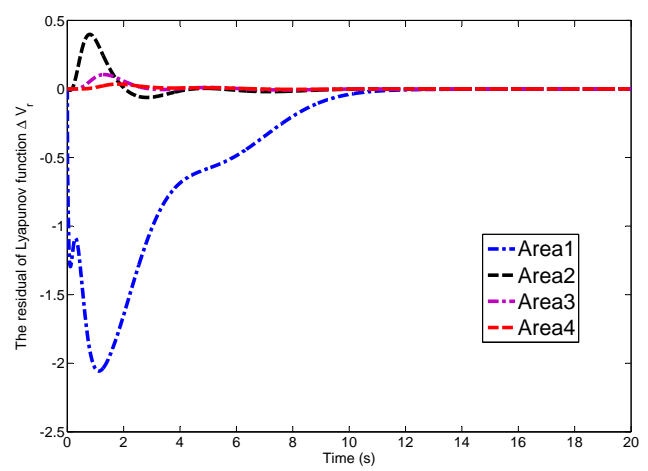

Fig. 6. Example 2 - case study 2: Evolution of $\Delta V_{r}=$ $V_{\text {coopArea } 1}-V_{\text {coop }}$ indicating that when higher priority is applied to Area1, its performance is better than in the equal priority situation at a cost of the reduction of its neighbors' performances (weak coupling) 
Case study 3 - Cooperative control with optimal priority:

With the coupling parameter remaining as $\beta=1$, we explore the performance of the network when the optimal priority matrix is applied. At this step, each element in the priority matrix is chosen between $\pi_{i}^{j} \in[0.1,10]$ in the matrix pencil optimization. The resulted optimal priority matrix is given by:

$$
\Pi=\left(\begin{array}{cccc}
9.25 & 4.67 & 0 & 0 \\
5.26 & 9.56 & 3.70 & 0 \\
0 & 9.42 & 9.64 & 2.48 \\
0 & 0 & 8.51 & 9.52
\end{array}\right)
$$

Similarly, the performance of the network is verified by looking at the behaviors of the Lyapunov functions of each area $V_{\text {coopOptimal }}$. $V_{\text {coopOptimal }}$ is compared to $V_{\text {coop }}$ obtained in section 5.2.1. Fig. 7 depicts the improvement of the behaviors of all four areas.

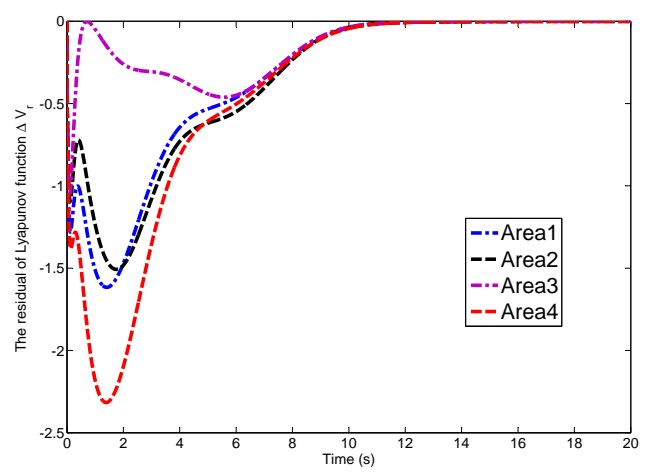

Fig. 7. Example 2 - case study 3: Evolution of $\Delta V_{r}=$ $V_{\text {coopOptimal }}-V_{\text {coop }}$ indicating that, in this case when optimal priority assignment is applied to all areas, their performances are all better than in the equal priority situation (weak coupling)

Case study 4 - Cooperative control under destabilizing interaction: In this simulation, the coupling parameter is set as $\beta=2$ and this changes the interaction between the areas to a destabilizing one. To stabilize the network despite of the destabilizing interconnection, we design the optimal priority matrix as presented in section 4 and each element is chosen between $[0.1,1000]$. The optimal priority matrix is:

$$
\Pi=\left(\begin{array}{cccc}
23.6 & 0.1 & 0 & 0 \\
1000 & 0.1 & 1000 & 0 \\
0 & 1000 & 0.1 & 1000 \\
0 & 0 & 1000 & 67.4
\end{array}\right)
$$

The initial conditions for all areas are zero and the evolutions of frequency deviation of each area under decentralized nominal control and cooperative control are shown in Fig. 8 and Fig. 9 respectively. The two figures show that the behaviors of the areas are unstable and unacceptable under decentralized nominal control while the cooperative control with the presence of optimal priority selection is able to stabilize all areas and the resulting frequency deviation is well controlled.
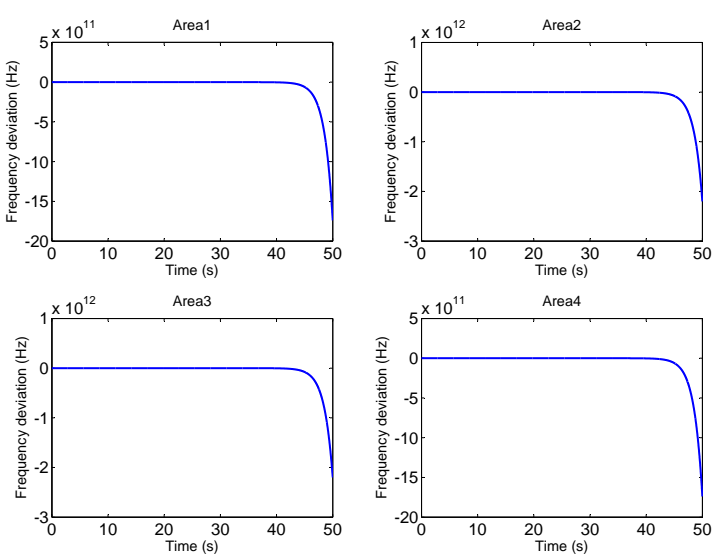

Fig. 8. Example 2 - case study 4: Frequency deviation without cooperative control in the case $\beta=2$ is used (destabilizing interaction)
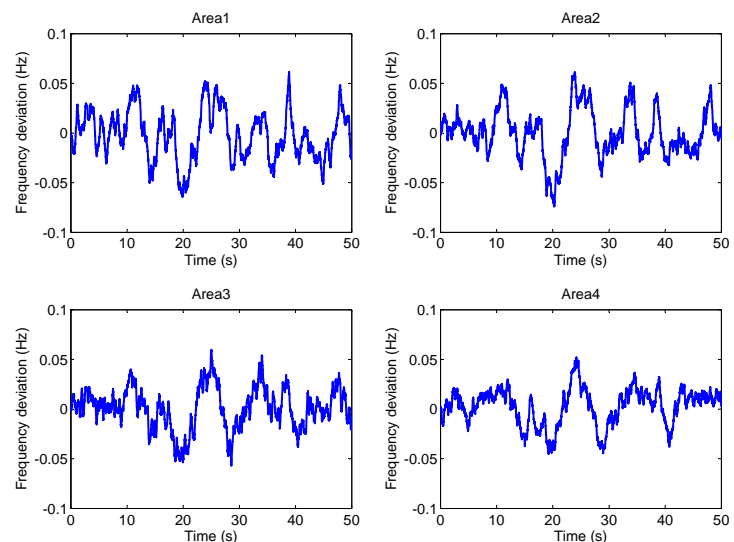

Fig. 9. Example 2 - case study 4: Frequency deviation with cooperative control (destabilizing interaction). The fluctuation is caused by a white noise to all states with covariance matrix $10^{-3} \times \mathbb{I}$.

\section{CONCLUSION}

In this paper, distributed control framework with limited communication has been proposed for interconnected subsystems with potentially destabilizing coupling. While each subsystem in the network has its own objective through an associated Lyapunov function, subsystems may cooperate when the integrity of the network is threatened. The choice of priority vector represents a standard matrix pencil optimization problem which can be solved offline using non convex optimization algorithms that may becomes interesting when high dimensional problems are tackled. The proposed framework is particularly attractive as a first step in the design of distributed control for nonlinear networks. Indeed, unlike many works where the degrees of freedom are represented by the linear feedback gain matrices which loose their relevance as soon as nonlinear dynamics are involved, the concept of relative priority coefficients can be used in both linear and nonlinear design [see (M. Alamir and Ding [2011])]. Moreover, the optimal 
relative priority set that is computed using the linearized model is likely to remain relevant even for the nonlinear network (at least as far as deviations are not very important). They can therefore be used as relevant design parameters for the nonlinear distributed control design. Investigating such situations and comparisons between such algorithms are currently under progress and represent obvious perspectives for the present paper.

\section{Appendix A. FINDING THE MATRICES}

As for the matrices $G_{j}^{i}, i=1, \ldots, N_{j}$, they are derived as follows (resulting in the equation (A.5) below) : Let us first recall the equation of subsystem $j$ :

$$
\dot{x}_{j}=A_{j}^{c l} x_{j}+\sum_{i \in \mathcal{I}_{j}^{\leftarrow}}\left(A_{i \rightarrow j} x_{i}+B_{i \rightarrow j} u_{i}\right)
$$

with $u_{i}=-K_{i} x_{i}+v_{i}$. Therefore equation (A.1) becomes:

$$
\dot{x}_{j}=A_{j}^{c l} x_{j}+\sum_{i \in \mathcal{I}_{j}^{\leftarrow}}\left[\left(A_{i \rightarrow j}-B_{i \rightarrow j} K_{i}\right) x_{i}+B_{i \rightarrow j} v_{i}\right]
$$

In order to build the dynamics that enables the additional control $v_{j}$ to be computed, subsystem $j$ assumes that the cooperative actions $v_{i}$ are zero for all subsystems $i$ s that are affected by $j\left(i \in \mathcal{I}_{j}^{\leftarrow}\right)$. The bi-directional interconnection relationship required in Assumption 1 is used now, and one can compare (A.2) and the first $n_{j}$ rows (the dynamics corresponding to $\dot{x}_{j}$ ) of (10) to find that:

$$
\pi_{j}^{i} \cdot G_{j}^{i} \cdot W_{i \succ j}^{T}=\left(A_{i \rightarrow j}-B_{i \rightarrow j} K_{i}\right) x_{i}
$$

and since $W_{i \longmapsto j}=2 x_{i}^{T} P_{i}$, one has:

$$
2 \pi_{j}^{i} \cdot G_{j}^{i} \cdot P_{i} \cdot x_{i}=\left(A_{i \rightarrow j}-B_{i \rightarrow j} K_{i}\right) x_{i}
$$

Therefore the matrices $G_{j}^{i}$ involved in the extended model (10) that is used by subsystem $j$ is given by:

$$
G_{j}^{i}=\frac{1}{2 \pi_{j}^{i}} \cdot\left(A_{i \rightarrow j}-B_{i \rightarrow j} K_{i}\right) \cdot\left(P_{i}\right)^{-1}
$$

\section{Appendix B. THE VALUES OF THE MATRICES USED IN EXAMPLE 1}

$$
\begin{gathered}
A_{1}=\left[\begin{array}{cc}
0.66 & 0.86 \\
0.56 & -0.16
\end{array}\right] \quad A_{2}=\left[\begin{array}{cc}
0.16 & 2.23 \\
1.59 & -0.79
\end{array}\right] \\
A_{3}=\left[\begin{array}{cc}
-0.55-1.85 \\
-1.17 & 0.64
\end{array}\right] \quad B_{1}=\left[\begin{array}{ll}
-0.02 & 1.84
\end{array}\right]^{T} \quad B_{2}=\left[\begin{array}{l}
-1.55 \\
-1.22
\end{array}\right] \\
B_{3}=\left[\begin{array}{cc}
-0.23 \\
0.09
\end{array}\right] \quad A_{2 \rightarrow 1}=\left[\begin{array}{cc}
1.36 & -0.49 \\
-1.99 & -0.28
\end{array}\right] \\
A_{3 \rightarrow 1}=\left[\begin{array}{ll}
1.46 & -0.90 \\
0.64 & 0.02
\end{array}\right] \\
B_{2 \rightarrow 1}=\left[\begin{array}{cc}
-0.20-0.77
\end{array}\right]^{T} \quad B_{3 \rightarrow 1}=\left[\begin{array}{ll}
1.09 & -0.05
\end{array}\right]^{T} \\
A_{1 \rightarrow 2}=\left[\begin{array}{cc}
0.87 & -0.30 \\
1.17 & 0.27
\end{array}\right] \quad A_{3 \rightarrow 2}=\left[\begin{array}{ll}
0.05 & 1.30 \\
0.05 & 1.80
\end{array}\right] \\
B_{1 \rightarrow 2}=\left[\begin{array}{lll}
-0.02-0.68
\end{array}\right]^{T} \quad B_{3 \rightarrow 2}=\left[\begin{array}{ll}
-0.09 & -0.09
\end{array}\right]^{T}
\end{gathered}
$$

$$
\begin{aligned}
& A_{1 \rightarrow 3}=\left[\begin{array}{cc}
1.06 & -1.16 \\
0.73 & -0.66
\end{array}\right] \quad A_{2 \rightarrow 3}=\left[\begin{array}{cc}
-0.09 & 0.71 \\
-0.97 & -1.36
\end{array}\right] \\
& B_{1 \rightarrow 3}=[-1.60-1.45]^{T} \quad B_{2 \rightarrow 3}=\left[\begin{array}{ll}
0.81 & 1.73
\end{array}\right]^{T}
\end{aligned}
$$

\section{Appendix C. THE DESCRIPTION OF THE} ELECTRICAL POWER NETWORKS

The network consists of four identical subsystems (areas) that are interconnected in a chain graph as shown in Fig. 4 . Each subsystem has 7 states: $\Delta P_{t i e_{i}}, \Delta f_{i}, \Delta P_{g_{i}}, \Delta x_{v g_{i}}$, $\Delta P_{E_{i}}, \Delta P_{H_{i}}$ and $U_{A R_{i}} . \Delta P_{t i e_{i}}$ is the tie-line power flow of subsystem $i . \Delta f_{i}$ is the frequency deviation. $\Delta P_{g_{i}}$ is the output of gas turbine generator while $\Delta x_{v g_{i}}$ is its input. $\Delta P_{E_{i}}, \Delta P_{H_{i}}$ are the outputs of the battery electric storage system and the electric water heater respectively. The regional demand is $A R_{i}=\Delta P_{t i e_{i}}-B_{i} \Delta f_{i}$ and we define $U_{A R_{i}}=\int A R_{i} d t$. The interconnected term $\Delta P_{t i e_{i}}$ of subsystem $i$ is expressed as $\Delta P_{t_{i e}}=T_{i j}\left(\Delta f_{j}-f_{i}\right)$ where $j$ stands for the interconnecting subsystem with $i$. $a_{g}, a_{E}$ and $a_{H}$ are chosen to satisfy the ratio of the capacity of the corresponding gas turbine, battery electric storage and electric water heater. Moreover, they also satisfy the following equality $a_{g}+a_{E}+a_{H}=1 . B_{i}, T_{i j}$ and $R_{g}$ are the frequency bias, synchronizing coefficient and regulation constant respectively. The state space models of each subsystem are given in the following form:

$$
\dot{x_{i}}=A_{i} x_{i}+B_{i} u_{i}+\omega+\beta \sum_{j \in \mathcal{I}_{i}^{\leftarrow}} A_{j \rightarrow i} x_{j}
$$

$\omega$ is a white noise added to all states (with covariance $10^{-3}$ in this paper). The states and the system matrices can be written as:

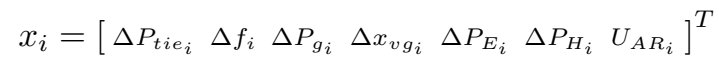

$$
B_{i}=\left[\begin{array}{ccccccc}
0 & 0 & 0 & 0 & 0 & 0 & 0 \\
0 & 0 & 0 & 0 & 0 & 0 & 0 \\
0 & 0 & 0 & 0 & 0 & 0 & 0 \\
0 & 0 & 0 & \frac{1}{T_{g_{i}}} & 0 & 0 & 0 \\
0 & 0 & 0 & 0 & \frac{1}{T_{E_{i}}} & 0 & 0 \\
0 & 0 & 0 & 0 & 0 & \frac{1}{T_{H_{i}}} & 0 \\
0 & 0 & 0 & 0 & 0 & 0 & 0
\end{array}\right]
$$

$$
A_{i}=\left[\begin{array}{ccccccc}
0 & -\sum_{j \in \mathcal{I}_{i}^{\leftarrow}} T_{i j} & 0 & 0 & 0 & 0 & 0 \\
\frac{1}{M_{i}} & -\frac{D}{M_{i}} & \frac{1}{M_{j}} & 0 & \frac{1}{M_{i}} & -\frac{1}{M_{i}} & 0 \\
0 & 0 & -\frac{1}{T_{d_{i}}} & \frac{1}{T_{d_{i}}} & 0 & 0 & 0 \\
0 & -\frac{1}{T_{g_{i}} R_{g_{i}}} & 0 & -\frac{1}{T_{g_{i}}} & 0 & 0 & \frac{a_{g} K_{i}}{T_{g}} \\
0 & -\frac{1}{T_{E_{i}} R_{g_{i}}} & 0 & 0 & -\frac{1}{T_{E_{i}}} & 0 & \frac{a_{g} K_{i}}{T_{E}} \\
0 & -\frac{1}{T_{H_{i}} R_{g_{i}}} & 0 & 0 & 0 & -\frac{1}{T_{H_{i}}} & \frac{a_{g} K_{i}}{T_{H}} \\
1 & -B_{i} & 0 & 0 & 0 & 0 & 0
\end{array}\right]
$$




$$
\begin{aligned}
A_{j \rightarrow i}= & {\left[\begin{array}{ccccccc}
0 & T_{i j} & 0 & 0 & 0 & 0 & 0 \\
0 & 0 & 0 & 0 & 0 & 0 & 0 \\
0 & 0 & 0 & 0 & 0 & 0 & 0 \\
0 & 0 & 0 & 0 & 0 & 0 & 0 \\
0 & 0 & 0 & 0 & 0 & 0 & 0 \\
0 & 0 & 0 & 0 & 0 & 0 & 0 \\
0 & 0 & 0 & 0 & 0 & 0 & 0
\end{array}\right], \quad j \in \mathcal{I}_{i}^{\leftarrow} } \\
& A_{j \rightarrow i}=\mathbb{O}
\end{aligned}
$$

The numerical values of the parameters are taken from (Namerikawa and Kato [2011]) and are shown on Table. C.

\begin{tabular}{lccc} 
Parameters & Symbol & Value & Unit \\
\hline inertia constant & $M$ & 0.20 & $p u M W \cdot s / H z$ \\
damping constant & $D$ & 0.26 & $p u M W / H z$ \\
governor time constant & $T_{g}$ & 0.20 & $s$ \\
gas turbine constant & $T_{d}$ & 5.0 & $s$ \\
BESS time constant & $T_{E}$ & 0.20 & $s$ \\
HP time constant & $T_{H}$ & 4.5 & $s$ \\
Regulation constant & $R_{g}$ & 2.5 & $\mathrm{~Hz} / \mathrm{pu} M W$ \\
Synchronizing coefficient & $T_{i j}$ & 0.50 & $p u M W$
\end{tabular}

Table C.1. Values of the parameters

\section{REFERENCES}

J.B. Rawlings A.N. Venkat, I.A. Hiskens and S.J. Wright. Distributed mpc strategies with application to power system automatic generation control. IEEE Transctions on Control System Technology, 16(6):1192-1206, 2008.

James B. Rawlings Stephen J. Wright Gabriele Pannocchia Brett T. Stewart, Aswin N. Venkat. Cooperative distributed model predictive control. Systems \&6 Control Letters, 59:460-469, 2010.

J.V. Burke, A.S. Lewis, and M.L. Overton. Optimizing matrix stability. Proceedings of the American Mathematical Society, 129(6):1635-1642, 2000.

E. Camponogara and L.B. de Oliveira. Distributed optimization for model predictive control of lineardynamic networks. IEEE Transactions on Systems, Man and Cybernetics, Part A: Systems and Humans, 39 (no.6)(6):1331-1338, 2009. doi: 10.1109/TSMCA.2009.2025507.

H. Cui and E.W. Jacobsen. Performance limitations in decentralized control. J. Proc. Control, 12:485-494, 2002.

W.B. Dunbar. Distributed receding horizon control of dynamically coupled nonlinear systems. 52(7):12491263, 2007. doi: 10.1109/TAC.2007.900828.

W.B. Dunbar and D.S. Caveney. Distributed receding horizon control of vehicle platoons: Stability and string stability. IEEE Transactions on Automatic Control, 57-3, 2012.

W.B. Dunbar and R.M. Murray. Distributed receding horizon control for multivehicle formation stabilization. Automatica, 42:549-558, 2006.

B.H. Krogh E. Camponogara, D. Jia and S. Talukdar. Distributed model predictive control. IEEE Control Systems Magazine, 22(1):44-52, 2002. doi: $10.1109 / 37.980246$.

M. Farina and R. Scattolini. Distributed predictive control: A non-cooperative algorithm with neighbor-toneighbor communication for linear systems. Automatica, 48:1088-1096, 2012.
E. Franco, L. Magni, T. Parisini, M. Polycarpou, and D. Raimondo. Cooperative constrained control of distributed agent with nonlinear dynamics and delayed information exchange: A stabilizing receding horizon approach. IEEE Trans. on Automatic Control, 53: 324-338, 2008.

Y.C. Ho. On centralized optimal control. IEEE Transactions on Automatic Control, 50(4):537-538, 2005.

D. Jia and B.H. Krogh. Distributed model predictive control. Proceedings of the American Control Conference, 2001.

A. Hably M. Alamir and H. Ding. A novel distributed nmpc control structure for partially cooperative systems under limited information sharing. In Proceedings of the 18th IFAC World Congress, 2011.

A.N. Michel and R.K. Miller. Stability analysis of discretetime interconnected systems via computer-generated lyapunov functions with applications to digital filters. IEEE Transactions on Circuits and Systems, 32-2, 1985.

T. Namerikawa and T. Kato. Distributed load frequency control of electrical power networks via iterative gradient methods. In 50th IEEE Conference on Decision and Control and European Control Conference, 2011.

A. Richards and J. How. A decentralized algorithm for robust constrained model predictive control. In Proceeding of the 2004 American Control Conference, 2004a.

A. Richards and J. How. Decentralized model predictive control of cooperating uavs. In 43rd IEEE Conference on Decision and Control, 2004b.

A. Richards and J.How. Robust distributed model predictive control. International Journal of control, 80(9): 1517-1531, 2007.

F. Borrelli J. Asgari T. Keviczky, P. Falcone and D. Hrovat. Predictive control approach to autonomous vehicle steering. In Proc. American Control Conf, 2006. doi: 10.1109/ACC.2006.1657458.

A. Song T. Li and S. Fei. Synchronization control for arrays of coupled discrete-time delayed cohengrossberg neural networks. Neurocomputing, 74:197-204, 2010. 